\title{
Effects of Single Administered Bofutsushosan-Composed Crude Drugs on Diabetic Serum Parameters in Streptozotocin-Induced Diabetic Mice
}

\author{
Qing Yu ${ }^{1}$, Tatsuo Takahashi ${ }^{1}$, Masaaki Nomura ${ }^{2}$, Mai Yasuda ${ }^{1}$, Kyoko Obatake-Ikeda ${ }^{3}$, \\ Shinjiro Kobayashi ${ }^{{ }^{*}}$ \\ ${ }^{1}$ Department of Clinical Pharmacy, Faculty of Pharmaceutical Sciences, Hokuriku University, \\ Kanazawa, Japan \\ ${ }^{2}$ Department of Education Center of Clinical Pharmacy, Faculty of Pharmaceutical Sciences, \\ Hokuriku University, Kanazawa, Japan \\ ${ }^{3}$ Department of Biochemistry, Faculty of Pharmaceutical Sciences, Hokuriku University, \\ Kanazawa, Japan \\ Email: ${ }^{*}$ s-kobayashi@hokuriku-u.ac.jp
}

Received December 20, 2012; revised January 26, 2013; accepted February 8, 2013

\begin{abstract}
The 18 crude drugs in Bofutsushosan (BOF: Pulvis ledebouriellae compositae: 防風通聖散) are separated into 6 groups such as diaphoretic, cathartic, antidote, antipyretic, neutralizer and diuretic groups. The effects of single administered $\mathrm{BOF}$ and composed crude drugs in 6 groups were investigated on the levels of diabetic parameters (serum glucose, insulin, triglyceride and cholesterol) in streptozotocin-induced diabetic mice. The anti-hyperglycemic action of BOF was depended on Ephedrae Herba, Saposhnikoviae Radix and Schizonepetae Spica in diaphoretic group, Forsythiae Fructus, Saposhnikoviae Radix, Schizonepetae Spica and Cnidii Rhizoma in antidote group, Scutellariae Radix, Gardeniae Fructus and Gypsum Fibrosum in antipyretic group and Paeoniae Radix in neutralizer group. In these crude drugs, Ephedrae Herba, Saposhnikoviae Radix, Schizonepetae Spica, Forsythiae Fructus, Scutellariae Radix, Gypsum Fibrosum and Paeoniae Radix increased serum insulin level, but Cnidii Rhizoma and Gardeniae Fructus did not affect serum insulin level. From these results, it suggested that anti-hyperglycemic action of BOF was through insulin-dependent and insulin independent manners. The lowering effect of BOF on serum triglyceride level was dependent on actions of Platycodi Radix in antidote and diuretic groups and Gardeniae Fructus in antipyretic group. The lowering effect of Gardeniae Fructus was parallel with its anti-hyperglycemic action. The lowering effect of BOF on high serum triglyceride level also included both direct action and indirect action. The reducing effect of BOF on serum cholesterol level was observed together with the actions of Ephedrae Herba and Zingiberis Rhizoma in diaphoretic group, Schizonepetae Spica in diaphoretic and antidote groups and Paeoniae Radix in neutralizer group. The lowering effects of Ephedrae Herba, Schizonepetae Spica and Paeoniae Radix on serum cholesterol level were parallel with their anti-hyperglycemic actions. Zingiberis Rhizoma in diaphoretic group might be direct reducing effect on serum cholesterol level but no serum glucose level. The Ephedrae Herba in diaphoretic group, Schizonepetae Spica in diaphoretic and antidote groups and Paeoniae Radix in neutralizer group might have reduced serum cholesterol level by reducing blood glucose level. From these results, composed crude drugs in 6 groups show various mechanisms in the action of BOF.
\end{abstract}

Keywords: Bofutsushosan (BOF); 6 Groups in BOF (Diaphoretic, Cathartic, Antidote, Antipyretic, Neutralizer and Diuretic Groups); Streptozotocin (STZ); Diabetic Serum Parameters; Anti-Hyperglycemia;

Anti-Hyperlipidemia

\section{Introduction}

Chinese medicine is used increasingly worldwide to treat chronic diseases, such as obesity, diabetes, hyperlipidemia and hypertension [1]. Metabolic syndrome is a direct consequence of diet specifically, intake of large

${ }^{*}$ Corresponding author. amounts of refined carbohydrates and sugars. Chinese medicine is an excellent system in complementary and alternative medicine. It holds great and unique potential in the management of metabolic syndrome, especially in the control of glucose and lipid metabolism [2].

Bofutsushosan (BOF), typical Chinese medicine, has been reported to improve atherosclerosis [3], obesity 
[4-6], hypertension, hyperglycemia [6], fatty liver [7,8] visceral fat and insulin resistance [9] in both clinical practice and animal examinations [5]. BOF has the potential to prevent adipogenesis in rat white adipocytes via modulation of gene expression levels [10]. Given by oral administered, BOF lowers fatty acid accumulation in the liver and reduces the levels of several plasma parameters correlated to obesity as well as body weight in mice fed a high-fat diet [8]. BOF produces a significant decrease in fat mass and weight compared with nontreated mice, without affecting the amount of food ingested in a study of obese mice [4]. In addition we have proved that BOF improved abnormal levels of serum glucose, insulin, triglyceride and cholesterol in STZ-diabetic mice [11]. BOF is promising for developing therapeutic medicine for metabolic diseases.

Streptozotocin (STZ) has been commonly used to induce a model of insulin-dependent diabetes mellitus (IDDM, type 1). STZ-induced diabetic mice have the features such as polydipsia, polyphagia, polyuria, dyslipidemia and hyperglycemia [12]. STZ also constructs a model of non-insulin-dependent diabetes mellitus (NIDDM, type 2) by its single administration. It has been reported that oral anti-hyperglycemic agents $[13,14]$ and Chinese medicines improved the hyperglycemia in STZ-diabetic model $[15,16]$. In the previous study [11], serum insulin level was increased by oral administration of BOF as well as glybenclamide in our STZ-diabetic mice. It suggested that our STZ-diabetic mice showed the feature of NIDDM.

BOF is consisted of Ephedrae Herba (麻黄), Saposhnikoviae Radix (防風), Zingiberis Rhizoma (生姜), Schizonepetae Spica (荊芥), Rhei Rhizoma (大黄), Natrium Sulfricum (芒硝), Glycyrrhizae Radix (甘草), Forsythiae Fructus (連堯羽), Platycodi Radix (桔梗), Cnidii Rhizoma (川芎), Scutellariae Radix (黄芩), Gardeniae Fructus (山栀子), Gypsum Fibrosum (石膏), Talcum (滑石), Angelicae Radix (当帰), Paeoniae Radix (苟薬), Atractyloidis Lanceae Rhizoma (蒼术), and Menthae Herba (薄荷) as shown in Table 1. These 18 composed crude drugs are separated into 6 groups such as diaphoretic, cathartic, antidote, antipyretic, neutralizer and diuretic groups. Diaphoretic group is consisted of Ephedrae Herba, Saposhnikoviae Radix, Zingiberis Rhizoma and Schizonepetae Spica to remove the diseases through perspiration from body surface. Cathartic group

Table 1. Dosaged of drugs composed in Bofutsushosan.

\begin{tabular}{|c|c|c|c|c|c|}
\hline & $\begin{array}{l}\text { Recovery rate of } \\
\text { extracted crude drugs \% } \\
(\mathrm{w} / \mathrm{w})\end{array}$ & $\begin{array}{l}\text { Dry weight of crude } \\
\text { drugs in BOF } \\
\text { (g) }\end{array}$ & $\begin{array}{l}\text { Amount of extracted } \\
\text { drugs in prescription } \\
\text { (g) }\end{array}$ & $\begin{array}{c}\text { Proportion of } \\
\text { Gardeniae Fructus }\end{array}$ & $\begin{array}{l}\text { Dosage of extracted } \\
\text { crude drugs } \\
(\mathrm{mg} / \mathrm{kg})\end{array}$ \\
\hline 1) Ephedrae Herba & 9.72 & 1.2 & 0.12 & 0.14 & 10 \\
\hline 2) Saposhnikoviae Radix & 9 & 1.2 & 0.11 & 0.13 & 10 \\
\hline 3) Zingiberis Rhizoma & 8.5 & 0.3 & 0.03 & 0.04 & 3 \\
\hline 4) Schizonepetae Spica & 7.02 & 1.2 & 0.08 & 0.10 & 10 \\
\hline 5) Rhei Rhizoma & 6.94 & 1.5 & 0.10 & 0.13 & 10 \\
\hline 6) Natrium Sulfricum & 96.7 & 0.7 & 0.68 & 0.71 & 100 \\
\hline 7) Glycyrrhizae Radix & 14.32 & 2.0 & 0.29 & 0.35 & 30 \\
\hline 8) Forsythiae Fructus & 31.76 & 1.2 & 0.38 & 0.47 & 30 \\
\hline 9) Platycodi Radix & 53 & 2.0 & 1.06 & 1.30 & 100 \\
\hline 10) Cnidii Rhizoma & 26.9 & 1.2 & 0.32 & 0.40 & 100 \\
\hline 11) Scutellariae Radix & 42.26 & 2.0 & 0.85 & 1.04 & 100 \\
\hline 12) Gardeniae Fructus & 67.7 & 1.2 & 0.81 & 1.00 & 100 \\
\hline 13) Gypsum Fibrosum & 2.12 & 2.0 & 0.04 & 0.05 & 3 \\
\hline 14) Talcum & 1.66 & 3.0 & 0.05 & 0.06 & 3 \\
\hline 15) Angelicae Radix & 32.6 & 1.2 & 0.39 & 0.48 & 30 \\
\hline 16) Paeoniae Radix & 30.6 & 1.2 & 0.37 & 0.45 & 30 \\
\hline $\begin{array}{l}\text { 17) Atractyloidis Lanceae } \\
\text { Rhizoma }\end{array}$ & 28.4 & 2.0 & 0.57 & 0.70 & 100 \\
\hline 18) Menthae Herba & 19.35 & 1.2 & 0.23 & 0.29 & 30 \\
\hline
\end{tabular}


is consisted of Rhei Rhizoma, Natrium Sulfricum and Glycyrrhizae Radix to soft intestinal contents, regulate intestinal movements and promote defecation. Antidote group is consisted of Forsythiae Fructus, Schizonepetae Spica, Saposhnikoviae Radix, Platycodi Radix and Cnidii Rhizoma to diminish toxins. Antipyretic group is consisted of Scutellariae Radix, Gardeniae Fructus, Gypsum Fibrosum and Talcum to eliminate internal thermal or inflammation. Neutralizer group is consisted of Angelicae Radix, Paeoniae Radix, Cnidii Rhizoma, Atractyloidis Lanceae Rhizoma and Menthae Herba to palliate and reduce the stimulation from the other drugs. Diuretic group is consisted of Paeoniae Radix, Atractyloidis Lanceae Rhizoma and Talcum to promote urination and remove of waste of body.

A lot of studies have demonstrated the effects of BOF on metabolic syndrome. However, there is no publication to investigate the effects of BOF on the diabetic parameters from the aspect of composed crude drugs in 6 groups. In this study, through using STZ-diabetic mice, we compared the effects of BOF and composed crude drugs on the levels of the diabetic parameters such as glucose, insulin, triglyceride and cholesterol in serum to deeply study the mechanisms of anti-hyperglycemia and anti-hyperlipidemia in BOF.

\section{Materials and Methods}

\subsection{Preparation of Streptozotocin-Diabetic Mice}

Fed male mice (ddY strain; 4 weeks of age; 16 - 20 g; Japan SLC, Shizuoka, Japan) were injected with a single dose $(150 \mathrm{mg} / \mathrm{kg}$ ) of STZ (Sigma, St. Louis, MO, USA) in saline into the tail vein. STZ-induced diabetic mice (7 - 8 weeks of age; blood glucose over $600 \mathrm{mg} / \mathrm{dl}$ ) were used for further experiments in 3 - 4 weeks after the injection of STZ. These mice were given by CRF-1 (Oriental Yeast Co., Tokyo, Japan) and water ad libitum and kept at $25^{\circ} \mathrm{C}-26^{\circ} \mathrm{C}$ with lights on from 7 a.m. to 7 p.m. $\mathrm{BOF}$, each 18 composed crude drug and water were administered intraperitoneally to 3 hours fasting STZ diabetic mice. The Ethics Review Committee for Animal Experimentation of Hokuriku University approved the experimental protocol.

\subsection{Preparation and Administration of Drugs}

BOF, Ephedrae Herba, Saposhnikoviae Radix, Zingiberis Rhizoma, Schizonepetae Spica, Rhei Rhizoma, Natrium Sulfricum, Glycyrrhizae Radix, Forsythiae Fructus, Platycodi Radix, Cnidii Rhizoma, Scutellariae Radix, Gardeniae Fructus, Gypsum Fibrosum, Talcum, Angelicae Radix, Paeoniae Radix, Atractyloidis Lanceae Rhizoma and Menthae Herba were purchased from Tsumura Co. (Tokyo) and extracted in 10 volumes of distilled wa- ter with an automatic extractor "Torobi" (Tochimoto, Osaka, Japan) for 1 hour. A water extract of the drug was filtered through a mesh (No. 42, Sanpo, Tokyo), lyophilized with a freeze-drier (DF-03G, ULVAC, Tokyo), and stored at $4^{\circ} \mathrm{C}[15,16]$. BOF, each composed crude drug extract and water were single administered intraperitoneally $(0.1 \mathrm{ml} / 10 \mathrm{~g}$ body weight $)$ into 3 hours fasting STZ-diabetic mice. Drug dosages were estimated on the basis of their recovery rates of crude drugs, dry weight of crude drugs in BOF, amount drug extracts. Table 1 showed recovery rate of extracted crude drugs, dry weight of crude drugs in BOF, amount of extracted drugs in prescription and proportion of Gardeniae Fructus. Dosages of extracted crude drugs were estimated by standardizing the dosage of Gardeniae Fructus $(100 \mathrm{mg} / \mathrm{k}$ g). In our previous study, $100 \mathrm{mg} / \mathrm{kg}$ was the effective dosage [11].

\subsection{Measurement of Glucose, Insulin, Triglyceride and Cholesterol Levels in Serum}

Blood was collected from the neck vein plexus of STZdiabetic mice before and 6 hours after single administration of drugs and water following 3 hours fasting. Blood samples were centrifuged at $8000 \mathrm{rpm}$ at $25^{\circ} \mathrm{C}$ for $5 \mathrm{~min}$. Serum glucose level of the supernatant was measured by the glucose oxidase method with a serum glucose monitor set (MEDISAFE MINI, Terumo, Tokyo). The fall \% of serum glucose (SG) was calculated as [SG (before drug treatment) - SG (after drug treatment)]/[SG (before drug treatment) -85$] \times 100$. The average SG of 3 hours fasting normal mice is $85 \mathrm{mg} / \mathrm{dl}[17,18]$. Serum insulin level was measured with a mouse ELISA kit for insulin (Morinaga, Yokohama, Japan), serum triglyceride and cholesterol levels were measured with ELISA kits (Wako, Osaka, Japan) at 6 hours after the single administration of drugs or water, respectively.

\subsection{Statistical Analyses}

All values were expressed as means \pm S.E.M. Differences between group data were evaluated by unpaired $t$-test at $p=0.05$ or 0.01 . A value of $p<0.05$ was considered statistically significant.

\section{Results}

\subsection{Effects of Composed Crude Drugs in 6 Groups of BOF on Serum Glucose Level in STZ-Diabetic Mice}

BOF $(300 \mathrm{mg} / \mathrm{kg})$ significantly lowered the high serum glucose level in STZ-diabetic mice. Effects of composed crude drugs in 6 groups of BOF, diaphoretic, cathartic, antidote, antipyretic, neutralizer and diuretic groups, were examined on the serum glucose level (Figure 1). 


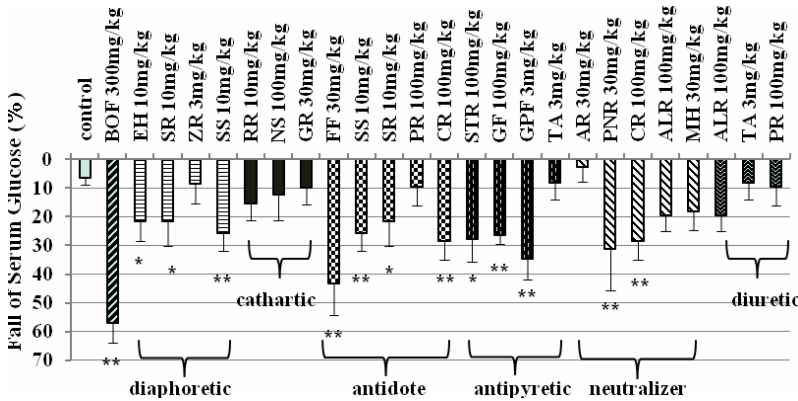

Figure 1. Effects of composed crude drugs in 6 groups of BOF on serum glucose level in STZ-diabetic mice. Serum glucose levels were measured before and 6 hours after i.p. administration of BOF and each composed crude drugs into 3 hours fasting STZ-diabetic mice. The values were expressed as means \pm S.E.M. of $5-15$ data. ${ }^{*} p<0.05,{ }^{* *} p<0.01$ : Significantly different from control water group. EH (Ephedrae Herba), SR (Saposhnikoviae Radix), ZR (Zingiberis Rhizoma), SS (Schizonepetae Spica), RR (Rhei Rhizoma), NS (Natrium Sulfricum), GR (Glycyrrhizae Radix), FF (Forsythiae Fructus), PR (Platycodi Radix), CR (Cnidii Rhizoma), STR (Scutellariae Radix), GF (Gardeniae Fructus), GPF (Gypsum Fibrosum), TA (Talcum), AR (Angelicae Radix), PNR (Paeoniae Radix), ALR (Atractyloidis Lanceae Rhizoma) and MH (Menthae Herba).

Ephedrae Herba (10 mg/kg), Saposhnikoviae Radix (10 $\mathrm{mg} / \mathrm{kg}$ ) and Schizonepetae Spica $(10 \mathrm{mg} / \mathrm{kg})$ in diaphoretic group significantly decreased $21.5 \%, 21.8 \%$ and $25.7 \%$ of serum glucose level respectively. However, Zingiberis Rhizoma (3 $\mathrm{mg} / \mathrm{kg}$ ) in diaphoretic group did not affect the high serum glucose level. Saposhnikoviae Radix and Schizonepetae Spica are not only in diaphoretic group but also in antidote group. In antidote group, Forsythiae Fructus $(30 \mathrm{mg} / \mathrm{kg})$ and Cnidii Rhizoma (100 $\mathrm{mg} / \mathrm{kg}$ ) also showed $43.4 \%$ and $28.3 \%$ fall of serum glucose level. But Platycodi Radix $(100 \mathrm{mg} / \mathrm{kg})$ in antidote group did not affect the high serum glucose level. In antipyretic group, Scutellariae Radix (100 mg/kg), Gardeniae Fructus $(100 \mathrm{mg} / \mathrm{kg})$ and Gypsum Fibrosum (3 $\mathrm{mg} / \mathrm{kg}$ ) showed $27.7 \%, 26.6 \%$ and $34.6 \%$ fall of serum glucose level. Cnidii Rhizoma is not only in antidote group but also in neutralizer group and showed $28.5 \%$ fall of serum glucose level. In neutralizer group, Paeoniae Radix $(30 \mathrm{mg} / \mathrm{kg}$ ) showed $31.1 \%$ fall of serum glucose level. Crude drugs in cathartic group and diuretic group did not affect the high level of serum glucose in STZ-diabetic mice.

\subsection{Effect of Composed Crude Drugs in 6 Groups of BOF, Which Lowered the High Serum Glucose Level, on Serum Insulin Level in STZ-Diabetic Mice}

BOF $(300 \mathrm{mg} / \mathrm{kg})$ significantly elevated the serum insulin level in STZ-diabetic mice. Effects of composed crude drugs in 6 groups of BOF, which lowered the high serum glucose level, were examined on the serum insulin level (Figure 2). Ephedrae Herba $(10 \mathrm{mg} / \mathrm{kg})$ in diaphoretic group, Saposhnikoviae Radix $(10 \mathrm{mg} / \mathrm{kg})$ and Schizonepetae Spica $(10 \mathrm{mg} / \mathrm{kg})$ in diaphoretic and antidote groups and Forsythiae Fructus $(30 \mathrm{mg} / \mathrm{kg})$ in antidote group significantly increased serum insulin level. Scutellariae Radix $(100 \mathrm{mg} / \mathrm{kg})$ and Gypsum Fibrosum (3 $\mathrm{mg} / \mathrm{kg}$ ) in antipyretic group and Paeoniae Radix (30 $\mathrm{mg} / \mathrm{kg}$ ) in neutralizer group also increased serum insulin level. However, Cnidii Rhizoma $(100 \mathrm{mg} / \mathrm{kg})$ in antidote group and Gardeniae Fructus $(100 \mathrm{mg} / \mathrm{kg})$ in antipyretic group did not affect serum insulin level, although reduced the high serum glucose level in STZ-diabetic mice.

\subsection{Effects of Composed Crude Drugs in 6 Groups of BOF on Serum Triglyceride Level in STZ-Diabetic Mice}

BOF $(300 \mathrm{mg} / \mathrm{kg})$ significantly lowered the high serum triglyceride level in STZ-diabetic mice. Effects of composed crude drugs in 6 groups of BOF, diaphoretic, cathartic, antidote, antipyretic, neutralizer and diuretic groups, were examined on serum triglyceride level (Figure 3). Platycodi Radix (100 mg/kg) in antidote group and diuretic group and Gardeniae Fructus $(100 \mathrm{mg} / \mathrm{kg})$ in antipyretic group significantly reduced the high serum triglyceride level. However, other 16 crude drugs did not affect the high level of serum triglyceride level in STZdiabetic mice.

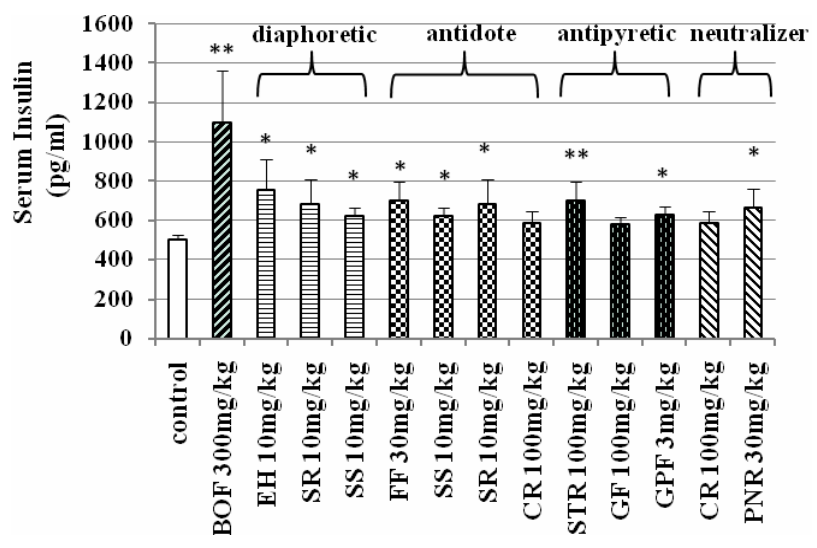

Figure 2. Effects of composed crude drugs in 6 groups of BOF, which lowered the high serum glucose level, on serum insulin level in STZ-diabetic mice. Serum insulin levels were measured at 6 hours after i.p. administration of BOF and each composed crude drugs which lowered the high serum glucose level into 3 hours fasting STZ-diabetic mice. The values were expressed as means \pm S.E.M. of 5 - 13 data. ${ }^{*} p<$ $0.05,{ }^{* *} p<0.01$ : Significantly different from control water group. EH (Ephedrae Herba), SR (Saposhnikoviae Radix), SS (Schizonepetae Spica), FF (Forsythiae Fructus), CR (Cnidii Rhizoma), STR (Scutellariae Radix), GF (Gardeniae Fructus), GPF (Gypsum Fibrosum), PNR (Paeoniae Radix). 


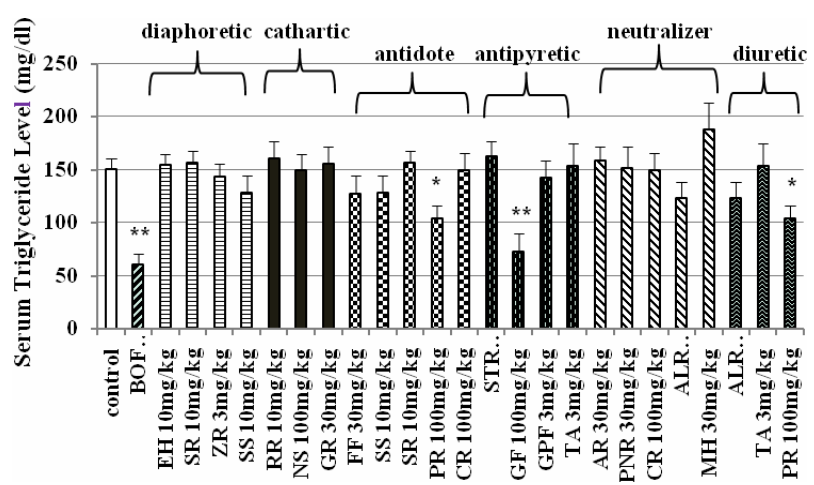

Figure 3. Effects of composed crude drugs in 6 groups of BOF on serum triglyceride level in STZ-diabetic mice. Serum triglyceride levels were measured at 6 hours after i.p. administration of BOF and each composed crude drugs into 3 hours fasting STZ-diabetic mice. The values were expressed as means \pm S.E.M. of 5 - 15 data. ${ }^{*} p<0.05,{ }^{* *} p<$ 0.01: Significantly different from the control water group. EH (Ephedrae Herba), SR (Saposhnikoviae Radix), ZR (Zingiberis Rhizoma), SS (Schizonepetae Spica), RR (Rhei Rhizoma), NS (Natrium Sulfricum), GR (Glycyrrhizae Radix), FF (Forsythiae Fructus), PR (Platycodi Radix), CR (Cnidii Rhizoma), STR (Scutellariae Radix), GF (Gardeniae Fructus), GPF (Gypsum Fibrosum), TA (Talcum), AR (Angelicae Radix), PNR (Paeoniae Radix), ALR (Atractyloidis Lanceae Rhizoma) and MH (Menthae Herba).

\subsection{Effects of Composed Crude Drugs in 6 Groups of BOF on Serum Cholesterol Level in STZ-Diabetic Mice}

BOF $(300 \mathrm{mg} / \mathrm{kg}$ ) significantly lowered the high serum cholesterol level in STZ-diabetic mice. Effects of composed crude drugs in 6 groups of BOF, diaphoretic, cathartic, antidote, antipyretic, neutralizer and diuretic groups, were examined on the high serum cholesterol level (Figure 4). Ephedrae Herba $(10 \mathrm{mg} / \mathrm{kg})$ and Zingiberis Rhizoma $(3 \mathrm{mg} / \mathrm{kg})$ in diaphoretic group, Schizonepetae Spica $(10 \mathrm{mg} / \mathrm{kg})$ in diaphoretic group and antidote group significantly lowered serum cholesterol level. Paeoniae Radix $(30 \mathrm{mg} / \mathrm{kg})$ in neutralizer group also lowered the high level of serum cholesterol in STZ-diabetic mice.

\section{Discussion}

STZ-induced diabetic model has been often used to the research about the diabetes mellitus according to the dosages used and experimental conditions [19]. Our STZdiabetic mice showed high serum glucose level, low serum insulin level and high serum triglyceride and cholesterol levels. BOF lowered high serum glucose level and elevated low serum insulin level [11], indicating that our STZ-diabetic mice had the ability to release insulin from pancreatic $\beta$ cell.

The effects of orally administered BOF on obesity [4-6], hypertension, hyperglycemia [6] and liver lipids $[7,8]$

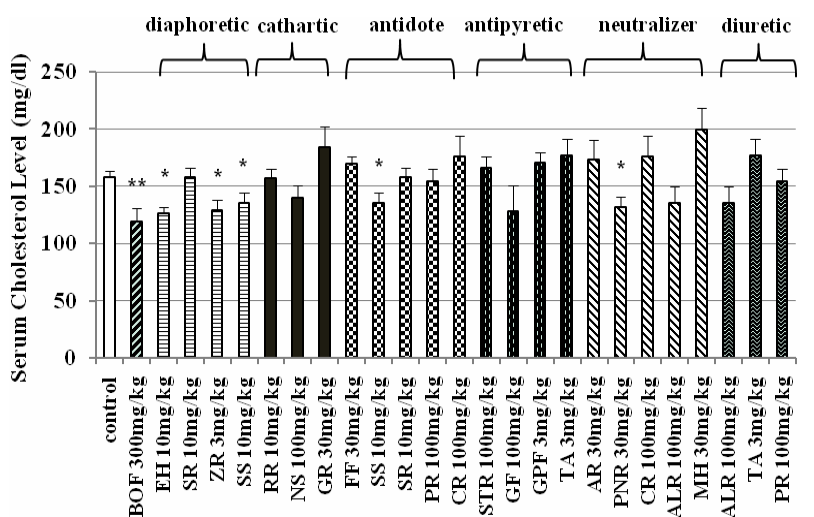

Figure 4. Effects of composed crude drugs in 6 groups of BOF on serum cholesterol level in STZ-diabetic mice. Serum cholesterol levels were measured at 6 hours after i.p. administration of BOF and each composed crude drugs into 3 hours fasting STZ-diabetic mice. The values were expressed as means \pm S.E.M. of 5 - 15 data. ${ }^{*} p<0.05,{ }^{* *} p<$ 0.01: Significantly different from the control water group. EH (Ephedrae Herba), SR (Saposhnikoviae Radix), ZR (Zingiberis Rhizoma), SS (Schizonepetae Spica), RR (Rhei Rhizoma), NS (Natrium Sulfricum), GR (Glycyrrhizae Radix), FF (Forsythiae Fructus), PR (Platycodi Radix), CR (Cnidii Rhizoma), STR (Scutellariae Radix), GF (Gardeniae Fructus), GPF (Gypsum Fibrosum), TA (Talcum), AR (Angelicae Radix), PNR (Paeoniae Radix), ALR (Atractyloidis Lanceae Rhizoma) and MH (Menthae Herba).

were certificated. Generally, oral administration method is often used when Chinese medicine is administered. However, in order to evaluate the drug evaluation with accurate amount and avoid the influence of digestion, we chose single intraperitoneal administration method. The effects of single intraperitoneal administration of BOF were the same as oral administered effects on diabetic parameters such as glucose, insulin, triglyceride and cholesterol levels in serum. High serum glucose level was improved by BOF. BOF also decreased high serum triglyceride and cholesterol levels in a dose-dependent manner in STZ-diabetic mice [11].

From the view of traditional Chinese medicine, diabetes is induced by toxin turned from extra nutrition. There are series of symptoms in diabetes, such as more thirty, more drink, more urine, high blood glucose level, hot feeling, etc. BOF is a typical Chinese medicine, which can discharge the extra toxins in the body. In this study we deeply investigated the respective efficacy of composed crude drugs in 6 groups of BOF, diaphoretic, cathartic, antidote, antipyretic, neutralizer and diuretic groups, on the parameters of diabetes.

It has been reported that BOF has the function on promoting the insulin secretion to improve high blood glucose level [6]. It is necessary to analyze which composed crude drugs were participated in the anti-hyperglycemic effect of BOF. Thus we studied to compare the actions of 
BOF for the diabetic parameters with the actions of composed crude drugs in 6 groups of BOF. Both of recovery rate and drug dosages of crude drugs in BOF and the anti-hyperglycemic actions of crude drugs were measured in STZ-diabetic mice. Ephedrae Herba, Saposhnikoviae Radix and Schizonepetae Spica in diaphoretic group reduced high serum glucose level and improved serum insulin level in STZ-diabetic mice. It implied that antihyperglycemic action of diaphoretic group was associated with an insulin-dependent mechanism. Saposhnikoviae Radix and Schizonepetae Spica are not only in diaphoretic group but also in antidote group. Antidote group can dispose the toxins in the body to remove the source of diseases. In antidote group, Forsythiae Fructus and Cnidii Rhizoma also lowered the high serum glucose level. Forsythiae Fructus increased serum insulin level. However, Cnidii Rhizoma did not increase serum insulin level although lowered the high serum glucose level. The reducing effect of Cnidii Rhizoma might be associated with an insulin-independent manner. Antipyretic group was used to solve the symptoms in diabetes such as thirsty and hot feeling [20]. In antipyretic group, excepting Talcum, Scutellariae Radix, Gardeniae Fructus and Gypsum Fibrosum all reduced high serum glucose level in STZ-diabetic mice. At the same time, Scutellariae Radix and Gypsum Fibrosum significantly increased serum insulin level, but Gardeniae Fructus did not affect serum insulin level. It implied that there were insulin-dependent and insulin-independent manners on the action of lowering high serum glucose level in the antipyretic group. Paeoniae Radix in neutralizer group also lowered high serum glucose level and improved serum insulin level. However the other crude drugs in neutralizer did not show the effect of lowering high serum glucose level in STZ-diabetic mice. From the results of anti-hyperglycemic actions and insulin-increasing actions of composed crude drugs in 6 groups of BOF, the anti-hyperglycemic action of BOF had two mechanisms such as in insulindependent and insulin-independent manners. Actions of Ephedrae Herba, Saposhnikoviae Radix, Schizonepetae Spica, Forsythiae Fructus, Scutellariae Radix, Gypsum Fibrosum and Paeoniae Radix were associated with an insulin-dependent mechanism. On the other hand, actions of Cnidii Rhizoma and Gardeniae Fructus were associated with an insulin-independent mechanism. We must explore the mechanism of Cnidii Rhizoma and Gardeniae Fructus on lowering high serum glucose level in STZ-induced diabetic mice.

BOF lowered high serum triglyceride level in STZdiabetic mice in a dose-dependent manner [11]. As the same with our single administration of BOF, Saito et al. [21] reported that BOF inhibited pancreatic lipase in vitro and suppressed the elevation of plasma triglyceride after oral administration of lipid emulsion. Morimoto et al. [5] also proved that BOF inhibited triglyceride synthesis in the liver. From our results, Platycodi Radix in antidote and diuretic groups reduced the high serum triglyceride level in STZ-diabetic mice, but other 16 crude drugs did not affect serum triglyceride level. It implied that effect of BOF on the high serum triglyceride level should be dependent on effects of Platycodi Radix and Gardeniae Fructus. It has been reported that Platycodi Radix worked as a peroxisome proliferator-activated receptor gamma (PPAR- $\gamma$ ) activator in liver, and decreased serum triglyceride storage [22,23]. The inhibitory effect of Platycodi Radix on serum triglyceride level might be a direct reducing effect on triglyceride, being non-dependent on its anti-hyperglycemic action. Gardeniae Fructus also significantly lowered serum triglyceride level in STZ-diabetic mice. This reducing effect might depend on geniposide, the main compound of Gardeniae Fructus. Geniposide had the effect on lowering the quantity of visceral fat and showed an alleviating effect on abnormal glucose/lipid metabolism [24]. The lowering effect of Gardeniae Fructus on serum triglyceride was parallel with its anti-hyperglycemic action. It suggested that the reducing effect of Gardeniae Fructus might be an indirect reducing effect on serum triglyceride, being related to its anti-hyperglycemic action. These results indicated that the lowering effect of single administered BOF on high serum triglyceride level in STZ-diabetic mice had direct reducing effect such as the action of Platycodi Radix and indirect reducing effect such as the action of Gardeniae Fructus.

BOF also significantly lowered serum cholesterol level in STZ-diabetic mice [11]. This lowering effect may be useful for preventive therapy in lifestyle-related diseases since the Guidelines from the National Cholesterol Education Program recommended reduction of LDL cholesterol levels as the primary goal in cardiovascular riskreduction therapy [25]. The study had been reported that orally administered BOF lowered total cholesterol and LDL cholesterol levels. We threw more light on the reducing effect of BOF on high serum cholesterol level in STZ-diabetic mice. Ephedrae Herba and Zingiberis Rhizoma in diaphoretic group, Schizonepetae Spica in diaphoretic and antidote group and Paeoniae Radix in neutralizer group significantly lowered the serum cholesterol level of STZ-diabetic mice. It has been reported that Zingiberis Rhizoma was able to lower the serum cholesterol level in hypercholesterolemia rabbit [26]. The reducing effect of Zingiberis Rhizoma on serum cholesterol level was not depended on its anti-hyperglycemic action. On the other hand, reducing effects of Ephedrae Herba, Schizonepetae Spica and Paeoniae Radix on serum cholesterol level were parallel with their reducing effects on high serum glucose level and increasing effects on serum insulin level in STZ-diabetic mice. These results indicated 
that effects of Ephedrae Herba, Schizonepetae Spica and Paeoniae Radix on high serum cholesterol level were associated with their effects on reduction of high serum glucose level and increase of serum insulin level. Their improving actions on abnormal glucose and insulin metabolism might cause reducing of acetyl-CoA. Acetyl-CoA was biochemical precursor of cholesterol. The excess of acetyl-CoA in STZ-diabetic mice causes an excess of hydroxymethylglutaryl-CoA reductase (HMG-CoA). HMG$\mathrm{CoA}$ reductase is a rate-limiting enzyme in the cholesterol biosynthetic pathway. Inhibition of HMG-CoA reductase decreases cholesterol synthesis. The inhibition of HMG-CoA reductase is very effective in lowering serum cholesterol and LDL in most of animal species, including humans [27]. All of these results suggest that BOF has two mechanisms for lowering effects on serum cholesterol level. One mechanism is the direct action of Zingiberis Rhizoma on cholesterol biosynthesis in the action of BOF. The other mechanism is associated with their anti-hyperglycemic actions, inducing by Ephedrae Herba, Schizonepetae Spica and Paeoniae Radix in the action of BOF.

\section{Conclusion}

The present study demonstrated that single administrated BOF and composed crude drugs were investigated on the levels of diabetic parameters (serum glucose, insulin, triglyceride and cholesterol) in STZ-diabetic mice. The anti-hyperglycemic action of BOF had two mechanisms: one was insulin-dependent mechanism such as actions of Ephedrae Herba, Saposhnikoviae Radix, Schizonepetae Spica Forsythiae Fructus, Scutellariae Radix, Gypsum Fibrosum and Paeoniae Radix. The other was insulinindependent mechanism such as actions of Cnidii Rhizoma and Gardeniae Fructus. Platycodi Radix in antidote and diuretic groups and Gardeniae Fructus in antipyretic group reduced the high serum triglyceride level in STZ-diabetic mice. The action of Platycodi Radix might be a direct reducing action on serum triglyceride but no anti-hyperglycemic action. Gardeniae Fructus might be related to its anti-hyperglycemic action, although Gardeniae Fructus did not release serum insulin level. Lowering effect of high serum triglyceride level in BOF also included both direct effect and indirect effect on decrease of serum triglyceride. Zingiberis Rhizoma in diaphoretic group might be direct reducing effect on serum cholesterol level but no serum glucose level. The Ephedrae Herba in diaphoretic group, Schizonepetae Spica in diaphoretic and antidote groups and Paeoniae Radix in neutralizer group might have reduced serum cholesterol level by reducing serum glucose level. From these results, composed crude drugs in 6 groups of BOF show various mechanisms in the actions of BOF.

\section{REFERENCES}

[1] S. Hasani-Ranjbar, B. Larijani and M. Abdollahi, "A Systematic Review of Iranian Medicinal Plants Useful in Diabetes Mellitus," Archives of Medical Science, Vol. 4, No. 3, 2008, pp. 285-292.

[2] J. Yin, H. Zhang and J. Ye, "Traditional Chinese Medicine in Treatment of Metabolic Syndrome," Endocrine, Metabolic \& Immune Disorders Drug Targets, Vol. 8, No. 2, 2008, pp. 99-111. doi:10.2174/187153008784534330

[3] K. Ohno, H. W. Chung, I. Maruyama and T. Tani, "Bofutsushosan, a Traditional Chinese Formulation, Prevents Intimal Thickening and Vascular Smooth Muscle Cell Proliferation Induced by Balloon Endothelial Denudation in Rats," Biological \& Pharmaceutical Bulletin, Vol. 28, No. 11, 2005, pp. 2162-2165. doi:10.1248/bpb.28.2162

[4] T. Yoshida, N. Sakane, Y. Wakabayashi, T. Umekawa and M. Kondo, "Thermogenic Antiobesity Effects of Bofutsushosan in MSG Obese Mice," International Journal of Obesity and Related Metabolic Disorders, Vol. 19, No. 10, 1995, pp. 717-722.

[5] Y. Morimoto, M. Sakata, A. Ono, T. Maegawa and S. Tajima, "Effects of Bofutsushosan, a Traditional Chinese Medicine, on Body Fat Accumulation in Fructose-Loaded Rats," Nippon Yakurigaku Zasshi, Vol. 117, No. 1, 2001, pp. 77-86. doi:10.1254/fpj.117.77

[6] Y. Morimoto, M. Sakata, A. Ohno, T. Maegawa and S. Tajima, "Effects of Byakkokaninjinto, Bofutsushosan and Goreisan on Blood Glucose Level, Water Intake and Urine Volume in KKAy Mice," Nippon Yakugaku Zasshi, Vol. 122, No. 2, 2002, pp. 163-168.

[7] S. Sakamoto, S. Takeshita, S. Sassa, S. Suzuki, Y. Ishikawa and H. Kudo, "Effects of Colestimide and/or Bofutsushosan on Plasma and Liver Lipids in Mice Fed a HighFat Diet," In Vivo, Vol. 19, No. 6, 2005, pp. 1029-1033.

[8] T. Nakayama, S. Suzuki, H. Kudo, S. Sassa, M. Nomura and S. Sakamoto, "Effects of Three Chinese Herbal Medicines on Plasma and Liver Lipids in Mice Fed a High-Fat Diet," Journal of Ethnopharmacology, Vol. 109, No. 2, 2007, pp. 236-240. doi:10.1016/j.jep.2006.07.041

[9] C. Hioki, K. Yoshimoto and T. Yoshida, "Efficacy of Bofutsushosan, an Oriental Herbal Medicine, in Obese Japanese Women with Impaired Glucose Tolerance," Clinical and Experimental Pharmacology and Physiology, Vol. 31, No. 9, 2004, pp. 614-619. doi:10.1111/j.1440-1681.2004.04056.x

[10] J. Yamakawa, I. Yasuhito, T. Fumihide, T. Takahashi and J. Yoshida, "The Kampo Medicines Orengedokuto, Bofutsushosan and Boiogito Have Different Activities to Regulate Gene Expressions in Differentiated Rat White Adipocytes: Comprehensive Analysis of Genetic Profiles," Biological \& Pharmaceutical Bulletin, Vol. 31, No. 11, 2008, pp. 2083-2089. doi:10.1248/bpb.31.2083

[11] Q. Yu, M. Yasuda, T. Takahashi, M. Nomura, N. Hagino and S. Kobayashi, "Effects of Bofutsushosan and Gardeniae Frutus on Diabetic Serum Parameters in Streptozotocin-Induced Diabetic Mice," Chinese Medicine, Vol. 2, No. 4, 2011, pp. 130-137. doi:10.4236/cm.2011.24022

[12] C. Rerup and F. Tarding, "Streptozotocin and Alloxan- 
Induced Diabetes in Mice," European Journal of Pharmacology, Vol. 7, No. 1, 1969, pp. 89-96. doi:10.1016/0014-2999(69)90169-1

[13] J. Movassat and B. Portha, "Beta-Cell Growth in the Neonatal Goto-Kakisaki Rat and Regeneration after Treatment with Streptozotocin at Birth," Diabetologia, Vol. 42, No. 9, 1999, pp. 1098-1106. doi:10.1007/s001250051277

[14] M. S. Gokhale, D. H. Shah, Z. Hakim, D. D. Santani and R. K.Goyal, "Effect of Chronic Treatment with Amlodipine in Non-Insulin-Dependent Diabetic Rats," Pharmacological Research, Vol. 37, No. 6, 1998, pp. 455-459. doi:10.1006/phrs.1998.0319

[15] N. Nakashima, I. Kimura and M. Kimura, "Isolation of Pseudoproto-Timosaponin AIII from Rhizomes of Anemarrhena Asphodeloides and Its Hypoglycemic Activity in Streptozotocin-Induced Diabetic Mice," Journal of Natural Products, Vol. 56, No. 3, 1993, pp. 345-350. doi:10.1021/np50093a006

[16] T. Miura, H. Toyoda, M. Miyake, E. Ishihara, M. Usami and K. Tanigawa, "Hypoglycemic Action of Stigma of Zea mays L. in Normal and Diabetic Mice," Natural Medicines, Vol. 50, No. 5, 1996, pp. 363-365.

[17] Y. Y. Liu, S. Kobayashi, T. Tsutsumi and H. Kontani, "Combined Effects of Stephania Radix and Astragali Radix in Antihyperglycemic Action of Boiogito (Fang-jihuang-qi-tang) in Streptozotocin-Induced Diabetic Mice," Journal of Traditional Medicines, Vol. 17, No. 6, 2000, pp. 253-260.

[18] T. Tsutsumi, S. Kobayashi, Y. Y. Liu and H. Kontani, "Anti-Hyperglycemic Effect of Fangchinoline Isolated from Stephania tetrandra Radix in Streptozotocin-Diabetic Mice," Biological \& Pharmaceutical Bulletin, Vol. 26, No. 3, 2003, pp. 313-317. doi:10.1248/bpb.26.313

[19] A. Junod, A. E. Lambert, L. Orci, R. Pictet, A. E. Gonet and A. E. Renold, "Studies of the Diabetogenic Action of Streptozotocin," Proceedings of the Society for Experimental Biology and Medicine, Vol. 126, No. 1, 1967, pp. 201-205.

[20] Y. Z. Zhang, L. M. Kuang, J. Zhou, M. Wang, H. Li and Q. Yi, "30 Case of Type II Diabetic Treated with The Method of Dissipating Heat Detoxifying," Guangming Journal of Chinese Medicine, vol. 23 No. 5, 2008, pp 632-634.
[21] M. Saito, T. Hamazaki, T. Tani and S. Watanabe, "Bofutsushosan, a Traditional Chinese Formulation, Inhibits Pancreatic Lipase Activity in Vitro and Suppresses the Elevation of Plasma Triacylglycerols after Oral Administration of Lipid Emulsion," Journal of Traditional Medicines, Vol. 22, No. 3, 2005, pp. 308-313.

[22] L. K. Han, Y. N. Zheng, B. J. Xu, H. Okuda and Y. Kimura, "Saponins from Platycodi Radix Ameliorate High Fat Diet-Induced Obesity in Mice," The Journal of Nutrition, Vol. 132, No. 8, 2002, pp. 2241-2245.

[23] D. Y. Kwon, Y. S. Kim, S. Y. Ryu, Y. H. Choi, M. R. Cha, H. J. Yang and S. Park, "Platyconic Acid, a Saponin from Platycodi Radix, Improves Glucose Homeostasis by Enhancing Insulin Sensitivity in Vitro and in Vivo," European Journal of Nutrition, Vol. 51, No. 5, 2011, pp. 529540.

[24] K. Kojima, T. Shimade, Y. Nagareda, M. Watanabe, J. Ishizaki, Y. Sai, K. Miyamoto and M. Aburada, "Preventive Effect of Geniposide on Metabollic Disease Status in Spontaneously Obese Type 2 Diabetic Mice and Free Fatty Acid-Treated HepG2 Cells," Biological \& Pharmaceutical Bulletin, Vol. 34, No. 10, 2011, pp. 1613-1618. doi: $10.1248 / \mathrm{bpb} .34 .1613$

[25] Expert Panel on Detection, Evaluation, and Treatment of High Blood Cholesterol in Adults, "Executive Summary of the 3rd Report of the National Cholesterol Education Program (NCEP) Expert Panel on Detection, Evaluation, and Treatment of High Blood Cholesterol in Adults (Adult Treatment Panel III)," The Journal of the American Medical Association, Vol. 285, No. 19, 2001, pp. 24862497. doi:10.1001/jama.285.19.2486

[26] U. Bhandari, J. N. Sharma and R. Zafar, "The Protective Action of Ethanolic Ginger (Zingber Officinale) Extract in Cholesterol Fed Rabbits," Journal of Ethnopharmacology, Vol. 61, No. 2, 1998, pp. 167-171. doi:10.1016/S0378-8741(98)00026-9

[27] D. Amin, S. K. Gustafson, J. M. Weinacht, S. A. Cornell, K. Neuenschwander, B. Kosmider, A. C. Scotese, J. R. Regan and M. H. Perrone, "RG 12561 (Dalvastatin): A Novel Synthetic Inhibitor of HMG-CoA Reductase and Cholesterol-Lowering Agent," Pharmacology, Vol. 46, No. 1, 1993, pp. 13-22. doi:10.1159/000139024 